\title{
Molecular modeling of a $\mathrm{T}$-cell receptor bound to a major histocompatibility complex molecule: Implications for $\mathrm{T}$-cell recognition
}

\author{
JUAN C. ALMAGRO, ${ }^{1}$ ENRIQUE VARGAS-MADRAZO, ${ }^{2}$ FRANCISCO LARA-OCHOA, \\ AND EDUARDO HORJALES ${ }^{1}$ \\ ${ }^{1}$ Instituto de Quimica, Universidad Nacional Autónoma de México, Circuito Exterior, Ciudad Universitaria, \\ C.P. 04510 , México, D.F. \\ ${ }^{2}$ Instituto de Investigaciones Biológicas, Universidad Veracruzana, Xalapa, Veracruz, México \\ (Received April 4, 1995; ACCePted June 28, 1995)
}

\begin{abstract}
The main functions of the T-cell receptor (TCR) involve its specific interaction with short and linear antigenic peptides bound to the major histocompatibility complex (MHC) molecules. In the absence of a 3D structure for TCR and for the TCR/peptide/MHC complex, several attempts to characterize the structural components of the $\mathrm{TCR} /$ peptide/MHC interaction have been made. However, this subject is still troublesome.

In this paper a computer-based 3D model for a TCR/peptide/MHC complex (5C.C7/moth cytochrome $c$ [MCC] peptide 93-103/I- $E^{k}$ ) was obtained. The complex surface shows a high complementarity between the $5 \mathrm{C}$.C 7 structure and the peptide/I- $E^{k}$ molecule. The mapping of residues involved in the TCR/peptide/MHC interaction shows close agreement with mutational experiments (Jorgensen JL, Reay PA, Ehrich EW, Davis MM, 1992b, Annu Rev Immunol 10:835-873). Moreover, the results are consistent with a recent variability analysis of TCR sequences using three variability indexes (Almagro JC, Zenteno-Cuevas R, Vargas-Madrazo E, Lara-Ochoa F, 1995b, Int $J$ Pept Protein Res 45:180-186). Accordingly, the 3D model of the 5C.C7/MCC peptide 93-103/I-E ${ }^{k}$ complex provides a framework to generate testable hypotheses about TCR recognition. Thus, starting from this model, the role played by each loop that forms the peptide/MHC binding site of the TCR is discussed.
\end{abstract}

Keywords: TCR/peptide/MHC interaction complex; variability analysis

The specificity of the immune system is related to two protein families: Igs and TCRs. Igs directly recognize, via their $V_{L}$ and $\mathrm{V}_{\mathrm{H}}$ domains, the 3D structure of native antigenic proteins in solution (Davies et al., 1990), whereas TCRs recognize, via their $\mathrm{V}_{\alpha}$ and $\mathrm{V}_{\beta}$ domains, short antigenic peptides bound to the MHC molecules (Davis, 1990). At present, significant advances have been made in the structural characterization of antigenic recognition mediated by Igs (Davies et al., 1990; Wilson \& Stanfield, 1993; Padlan, 1994). In contrast, the nature of the interaction between TCRs and peptide-MHC complexes is poorly understood (Jorgensen et al., 1992b). This is partly due to the

Reprint requests to: Juan C. Almagro, Instituto de Quimica, Universidad Nacional Autónoma de México, Circuito Exterior, Ciudad Universitaria, C.P. 04510, México, D.F.; e-mail: almagro@vincent.iquimica. unam.mx.

Abbreviations: Ig, immunoglobulin; TCR, T-cell receptor; MHC, major histocompatibility complex; MCC, moth cytochrome $c$; PDB, Brookhaven Protein Data Bank; $V$, variable domain; $V_{L}$, variable domain of light chain; $V_{H}$, variable domain of heavy chain; $V_{\alpha}$, variable domain of $\alpha$ chain; $V_{\beta}$, variable domain of $\beta$ chain. lack of an experimental 3D structure for TCRs and for TCR/ peptide/MHC complexes.

In the absence of 3D structures for TCRs, sequence analysis of their $\mathrm{V}$ domains shows that the $\mathrm{V}_{\alpha} / \mathrm{V}_{\beta}$ dimer has a framework structure that is very close to the Fv fragment of Igs (Novotny et al., 1986; Chothia et al., 1988). Further comparison has structurally assigned the six hypervariable loops that form the antigen-binding site of Igs to the binding site of TCRs (Chothia et al., 1988), termed $\alpha 1, \alpha 2$, and $\alpha 3$ for the $\mathrm{V}_{\alpha}$, and $\beta 1, \beta 2$, and $\beta 3$ for the $\mathrm{V}_{\beta}$ in TCRs.

Genetically, $\alpha 1$ and $\alpha 2$ are encoded by the $\mathrm{V}_{\alpha}$ gene, whereas $\alpha 3$ is produced by the recombination of an additional gene segment: $J \alpha$ (Davis, 1990). In a similar way $\beta 1$ and $\beta 2$ are encoded by the $V_{\beta}$ gene, whereas $\beta 3$ is a result of the recombination of two additional gene segments: $\mathrm{D}$ and $\mathrm{J} \beta$ (Davis, 1990). Based on the fact that $\alpha 3$ and $\beta 3$ concentrate the genetic diversity of TCRs on the one hand, and the similarity between TCRs and Igs on the other, several hypothetical models for the TCR/peptide/MHC interaction have been proposed (Chothia et al., 1988; Davis \& Bjorkman, 1988; Claverie et al., 1989). These models 
predict that $\alpha 1, \alpha 2, \beta 1$, and $\beta 2$ recognize the $\alpha$-helices of $M H C$ molecules, whereas $\alpha 3$ and $\beta 3$ interact with the antigenic peptide (Chothia et al., 1988; Davis \& Bjorkman, 1988; Claverie et al., 1989).

The above models, for their generality, have been very useful for thinking about the evolution of the immune receptors (Davis \& Bjorkman, 1988; Claverie et al., 1989; Davis, 1990), for explaining overall features of the TCR/peptide/MHC interaction (Jorgensen et al., 1992b), and for suggesting experiments (Ajitkumar et al., 1988; Nalefski et al., 1992; Patten et al., 1993; Lone et al., 1994; Katayama et al., 1995). However, in their current form, they may not be sufficiently detailed to delimit which segments of the helical regions of MHC molecules interact with the TCR (Prochnicka-Chalufour et al., 1991; Almagro et al., $1995 b)$. Consequently, they do not assign a role for the $\alpha 1, \alpha 2$, $\beta 1$, and $\beta 2$ in the TCR-MHC interaction.

In Igs as well as in TCRs, it has been proposed that the most variable regions of their $\mathrm{V}$ domains must be placed in those loops that structurally form the binding site (Kabat et al., 1991). However, the precise identification of the hypervariable regions within the $\mathrm{V}_{\alpha}$ and $\mathrm{V}_{\beta}$ domains using the classical variability measure (Kabat variability index [Wu \& Kabat, 1970]) have been troublesome in all the TCR loops, except for $\alpha 3$ and $\beta 3$ (Kabat et al., 1991). Failure to identify the hypervariable regions in $\alpha 1$, $\alpha 2, \beta 1$, and $\beta 2$ of TCRs by the Kabat index is a consequence of the absence of dominant peaks of variability in the amino acid sequences encoded by the $V_{\alpha}$ and $V_{b}$ gen segments (Kabat et al., 1991). In order to solve this difficulty, three variability indexes followed by a Fourier filtering have been recently used (Almagro et al., 1995b). This methodology unambiguously delineates hypervariable regions at the $\alpha 2$ and $\beta 2$ loops together with the known hypervariable regions placed at the $\alpha 3$ and $\beta 3$ loops. However, the regions corresponding to $\alpha 1$ and $\beta 1$ have been found to be relatively conserved (Almagro et al., 1995b). These results suggest that $\alpha 1$ and $\beta 1$ could have a different role with respect to $\alpha 2$ and $\beta 2$ in the interaction with the $\mathrm{MHC}$ molecules (Almagro et al., 1995b).

In the last few years, increasing experimental evidence has accumulated for the interaction between TCR and peptide/MHC complexes (Jorgensen et al., 1992a; Nalefski et al,, 1992; Patten et al., 1993; DiGiusto \& Palmer, 1994; Lone et al., 1994; Katayama et al., 1995). One of the best-studied experimental systems is the T-cell response to different MCC peptides bound to the I- $E^{k}$ molecules (Hedrick et al., 1988; Jorgensen et al., 1992a; Patten et al., 1993; Katayama et al., 1995). In this system, the correlation of TCR gene segment usage with variants of the C-terminal peptide from MCC demonstrated that charge substitutions on different residues of the peptide elicit opposite charges in residues of $\alpha 3$ or $\beta 3$ of TCRs (Jorgensen et al., 1992a). This result implies a particular positioning on the peptide/MHC structure for the TCR. Therefore, the use of this experimental information allows us to refine the TCR/peptide/ MHC interaction models proposed at present in order to understand the role played by $\alpha 1, \alpha 2, \beta 1$, and $\beta 2$ in the MHC interaction.

Thus, for this paper a computer-based 3D model for the 5C.C7 sequence of TCRs in complex with peptide 93-103 of MCC (MCC [93-103]) and the I-E ${ }^{k}$ molecule was obtained. In the light shed by this atomic model, the structural role played by each of the loops that form the binding site of the TCR molecule in the interaction with peptide/MHC complex is discussed.

\section{Results}

\section{The atomic model of the TCR molecule}

The 3D structure for the 5C.C7 sequence was obtained by starting from the assumption that the $\mathrm{V}_{\alpha} / \mathrm{V}_{\beta}$ dimer of TCRs has the typical fold of an Fv fragment of Ig (Novotny et al., 1986; Chothia et al., 1988). In Igs, the Fv fragment is composed of a large conserved framework of $\beta$-sheets and six hypervariable loops denoted L1, L2, and $\mathrm{L} 3$ for $\mathrm{V}_{\mathrm{L}}$ and $\mathrm{H} 1, \mathrm{H} 2$, and $\mathrm{H} 3$ for $\mathrm{V}_{\mathrm{H}}$ (Wu \& Kabat, 1970; Poljak et al., 1973). Structural analysis of the binding site of Igs revealed that there are a small number of main-chain conformations found for five of the six hypervariable loops, termed canonical structures (Chothia \& Lesk, 1987). A canonical structure is determined (1) by the loop size and (2) by the presence of certain residues at key positions in both the hypervariable loops and the framework region (Chothia \& Lesk, 1987; Chothia et al., 1989; Tramontano et al., 1990). Molecular modeling procedures based on these rules have been demonstrated to be capable of correctly predicting the antigen-binding sites of different Fv fragments in advance of their experimental determination (Chothia et al., 1989).

Therefore, the framework of $5 \mathrm{C} . \mathrm{C} 7$ sequence was modeled by using as reference the coordinates of the D1.3 Fv fragment (Bhat et al., 1990), one of the best-resolved and best-refined FV structures (1.8 $\AA$ resolution and an $R$-value of 0.158 ). The alignment between the reference and $5 \mathrm{C} . \mathrm{C} 7$ sequence was made taking into account the amino acid pattern at those 40 positions proposed as crucial to maintain the conserved features of the Fv framework (Chothia et al., 1988) (Fig. 1).

Having modeled the framework of the 5 C.C7 sequence, the possibility of assembling the antigen-binding site based on coordinates from canonical structures was investigated. To achieve this, the size of canonical structures described for Igs was compared to the size of those loops that form the binding site of the 5C.C7 sequence. Three of the six loops, $\alpha 1, \beta 1$, and $\beta 2$ were found to have the proper size for canonical structures and consequently were built using this modeling procedure. The rest of the loops $(\alpha 2, \alpha 3$, and $\beta 3)$ do not have the appropriate size for a canonical structure as described at present. Thus, $\alpha 3$ and $\beta 3$ were modeled starting from coordinates of Ig loops found in a database of Ig structures by a loop search procedure. In Figure 2, the resulting five loops of 5C.C7 after molecular dynamics refinement of the complete structure (see the Materials and methods) are shown. In the case of $\alpha 2$, no appropriate loops were found in the Ig database. This loop was modeled as a deletion of two residues of $\mathbf{L} 2$ in the reference structure (see the Materials and methods).

Among the loops modeled with canonical structures, the coordinates of L1 type 1 were assigned to $\alpha 1$. This loop has residues different from those that stabilize the canonical conformation: Asp instead of lle at position 1, Phe instead of Ala at position 24, Val instead of Leu at position 32, Ser instead of Tyr at position 73, and Met instead Val at position 28 (numbering as in Fig. 1). However, after doing the molecular refinement of the structure, the results show that these residues are mutually compensated to fit the characteristic packing of the canonical structure Ll type 1 in $\alpha 1$ (see Fig. 2). This agrees with Ganju et al. (1992) modeling the binding site of the TCR sequence RFL8.3 based on the canonical structure motif.

The other loop modeled with atomic coordinates of a canonical structure was $\beta 1$. This loop is two residues shorter than the 


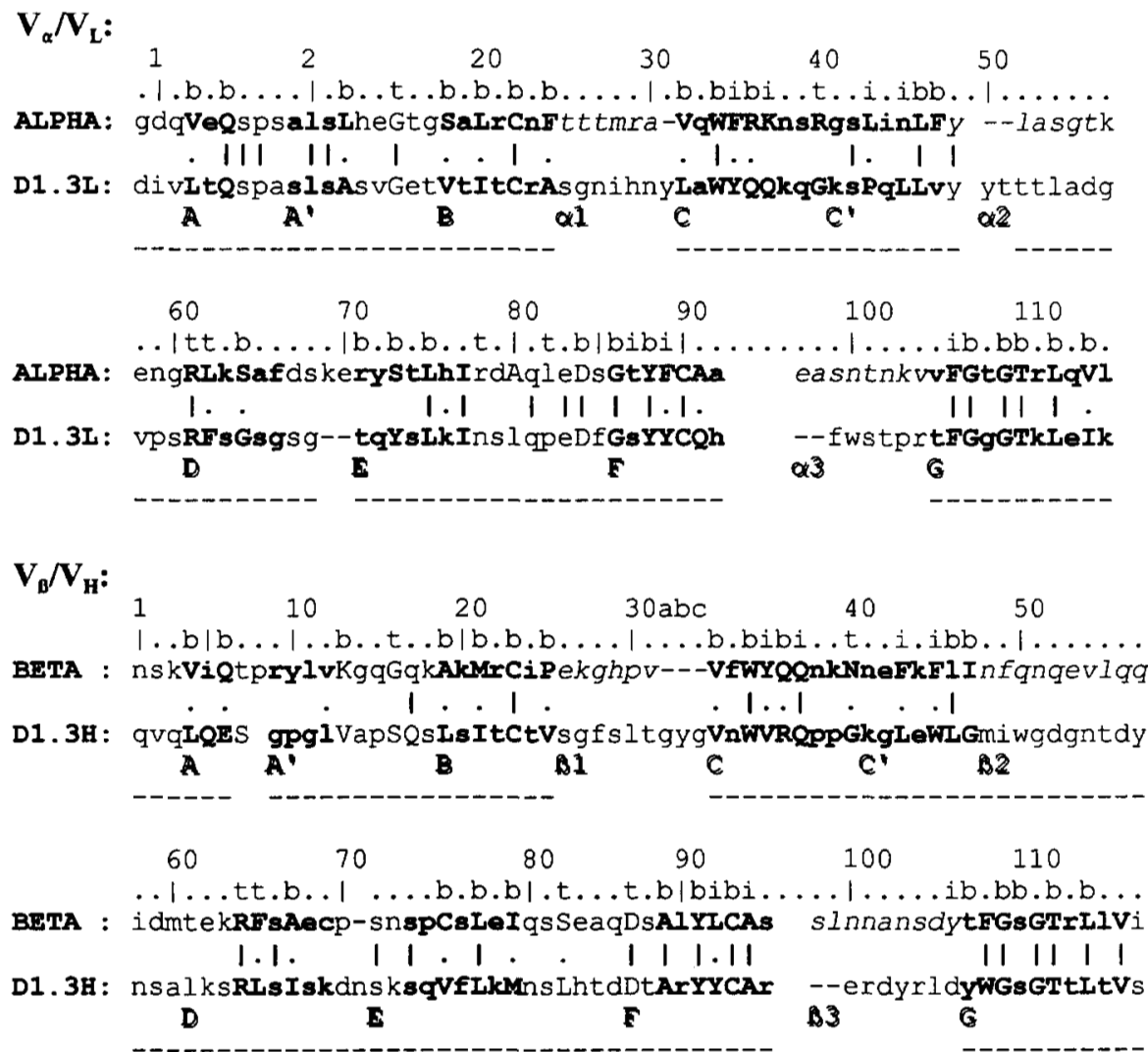

Fig. 1. Alignment between the reference (D1.3) and the TCR (5C.C7) sequence. Upper part of the figure, $\mathrm{V}_{\mathrm{L}} / \mathrm{V}_{\alpha}$ domains; lower part, $V_{H} / V_{\beta}$. Within residue numbering those residues that determine the Igfold (Chothia et al., 1988); b, buried in $\beta$ sheets, $t$, residues in turns, $\mathrm{i}$, at the interface between domains. In the sequences, letters in bold show the conserved $\beta$-sheet framework, letters in italics represent the hypervariable loops. Dotted lines under the TCR sequence indicate framework residues with backbone coordinates assigned using the reference structure. canonical structures described for H1 (Chothia \& Lesk, 1987; Chothia et al., 1992). However, this loop is equal in size to $\alpha 1$. Given this observation, along with the fact that $V_{\alpha}$ and $V_{\beta}$ possess features that are similar to both Ig domains, $V_{L}$ and $V_{H}$ (Chothia et al., 1988), $\beta 1$ was built with the canonical structure L1 type 1 also. However, at position 29 within the loop, $\beta 1$ has a large polar residue (histidine) differing from those residues (small hydrophobic) described as key to maintaining this canonical structure (Chothia \& Lesk, 1987; Chothia et al., 1989). An analysis of the refined structure (see Fig. 2) shows that, as in $\alpha 1$, mutations in residues of the framework can pack this canonical structure in $\mathrm{V}_{\beta}$. As can be seen from Figure 2, His at position 29 is stabilized by a hydrogen bond with Ser at framework position 94 of $V_{\beta}$. A study of the $V_{\beta}$ sequences has acknowledged this finding. In the compilation of $V_{\beta}$ sequences made by Chothia et al. (1988), this loop size is the most abundant within the sequences (roughly $90 \%$ ). Except for four, all sequences of this size have histidine at position 29 . Serine also is highly conserved at position 94 among the sequences with this loop size. Thus, the conservation of serine at position 94 brings forth a structural context that fits histidine as the anchor residue of the canonical structure $\mathrm{L} 1$ type 1 in $\mathrm{V}_{\beta}$.

The third 5C.C7 loop built with a canonical structure was $\beta 2$. This loop has the same size as the canonical structure $\mathrm{H} 2$ type 1 (Chothia \& Lesk, 1987). This canonical structure has Gly at positions 55 (Chothia et al., 1989) (position 53 of D1.3 $\mathrm{V}_{\mathrm{H}}$ in Fig. 1). Residue 53 is a Glu in the 5C.C7 loop, which produces a slight distortion of the conformation after the dynamical refinement (see Fig. 2).
For those loops with modeling not based on canonical structures ( $\alpha 3$ and $\beta 3$ ), the RMS differences between the $\mathrm{C} \alpha$ atoms of the X-ray coordinates used as reference structure and the final modeled structure were $0.57 \AA$ for $\alpha 3$ and $1.36 \AA$ for $\beta 3$. The differences show that these loops can be accommodated in the 5C.C7 framework. This result is consistent with the assessment of the final $5 \mathrm{C} . \mathrm{C} 7$ structure quality by the 3D-profile method (Lüthy et al., 1992). The structure has no regions scoring less than 0 (Fig. 3), and the global score of each $V$ domain of the TCR is comparable with global scores of X-ray structures of $\sim 100$ amino acid lengths (Lüthy et al., 1992). Furthermore, the $\alpha 2$ loop modeled as a deletion of two residues of $L 2$ in the reference structure shows a high score.

Finally, in order to assure the model's stability, the 5C.C7 structure was subjected to $30 \mathrm{ps}$ of molecular dynamic simulation at $300 \mathrm{~K}$ in a $4-\AA$ shell water. The RMS deviations over the $\mathrm{C} \alpha$ atoms between the framework of the starting structure and an average structure of the last 20 ps of simulation was of $1.41 \AA$. This RMS deviation is comparable with the correspondent value obtained among known X-ray structures of Igs (Chothia \& Lesk, 1987), as well as differences between an X-ray structure and an average structure of 40 ps of dynamical simulation for an $\mathrm{Fv}$ fragment in similar simulation conditions (Almagro et al., 1995a). In the loops that form the binding site, the RMS differences from the $\mathrm{C} \alpha$ atoms with respect to the 5C.C7 model structure are similar to the differences from the framework when compared with the initial model and the average structure. Thus, together with the 3D-profile method this test indicates that the $5 \mathrm{C} . \mathrm{C} 7$ model provides a good starting 


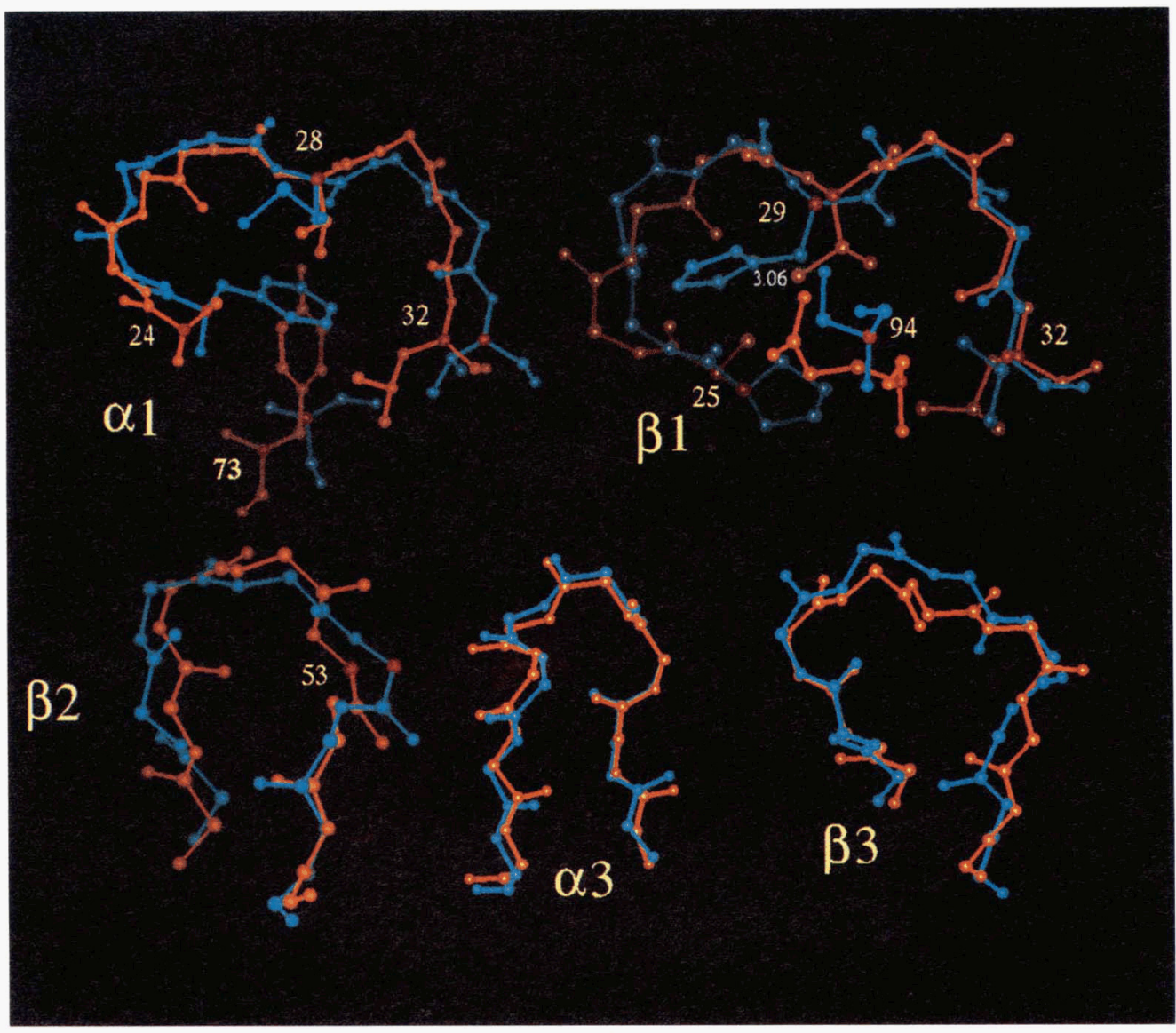

Fig. 2. Comparison of the loops that form the antigen-binding site in the $5 \mathrm{C} . \mathrm{C} 7$ molecule (blue) after molecular refinement and the X-ray structures used as reference in the molecular modeling procedure (orange). A red sphere on the C $\alpha$ atoms highlights those residues that determine the canonical structures in the Ig hypervariable loops. $\alpha 2$ is not depicted in the figure because this loop has no equivalent in the Igs of known structure. In $\alpha 1$ the residue at position 1 is not represented in the figure. In the $\beta 1$ loop the distance of the possible hydrogen bond between the His-29 and Ser-94 is shown in white.

structure to simulate the interaction with the MCC $(93-103) /$ I-E ${ }^{k}$ complex.

\section{The atomic model of the TCR/peptide/MHC complex}

The 3D model of the MCC peptide 93-103 in complex with the I-E ${ }^{k}$ molecule was obtained by taking advantage of the recently resolved X-ray crystallographic coordinates of the HLA-DR1 molecule in complex with the influenza hemagglutinin peptide 306-318 at $2.75 \AA$ (Stern et al., 1994). In Figure 4, the sequence alignment of the $\alpha 1$ and $\beta 1$ domains of HLA-DR 1 and $I-E^{k}$ is shown.

The alignment has no insertions or deletions. In addition, the sequences have identities of $78 \%$ for the $\alpha 1$ domain and $67 \%$ for the $\beta 1$ domain. Consequently, the direct mutations in side chains would produce a good starting model for further refinement by molecular dynamics. Starting from this structure and the 5C.C7 structure described in the above section, the atomic model for the 5C.C7/MCC (93-103)/I- $\mathrm{E}^{\mathrm{k}}$ complex was obtained.

The complex was built by manual orientation of the 5C.C7 molecule upon the $\mathrm{MCC}(93-103) / \mathrm{I}-\mathrm{E}^{\mathrm{k}}$ complex until the distance among the residues identified being in contact by muta- tional studies was optimal (Glu-97 of $\alpha 3$ and Asn-100 of $\beta 3$ with Lys-99 and Thr-102 in MCC, respectively [Jorgensen et al., 1992a]). Furthermore, the total energy upon the complex formation in each orientation was evaluated by calculating the van der Waals and electrostatic intermolecular interactions. The solvent-accessible surface of the loops that forms the binding site of TCR and the peptide/ $\alpha$-helices of MHC was visually analyzed during graphic manual changes. This procedure gives an additional criterion concerning surface complementarity.

In the conformation with minimal interaction energy and maximum complementarity between 5C.C7 and MCC(93-103)/ $I-E^{k}$, the complex was subjected to molecular dynamic refinement in a water shell. No major conformational changes were observed between the single molecules and the molecules of the complex, which indicate that the complex refinement only introduced local optimization of the side-chain interactions. The resulting structure is shown in Figure 5 and Kinemage 1.

Analysis of the complex interface shows that it is extended over a large region with maximum dimensions of $25 \AA \times 20 \AA$, similar to those predicted by mutagenesis studies (Ajitkumar et al., 1988). The solvent-accessible surface buried in complex formation was estimated in $\sim 900 \AA^{2}$ for $\mathrm{MCC} / \mathrm{I}-\mathrm{E}^{\mathrm{k}}$ and 


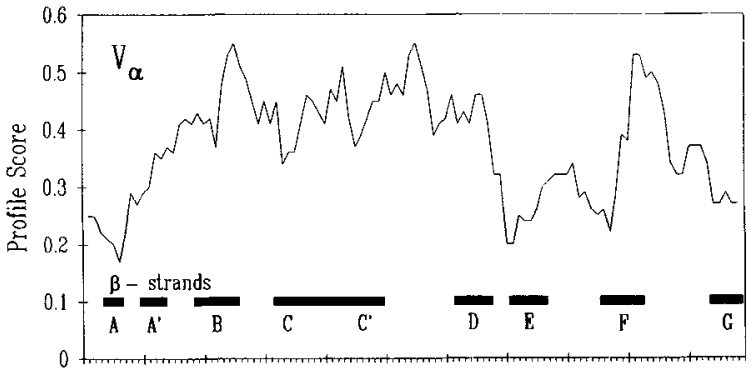

Residue

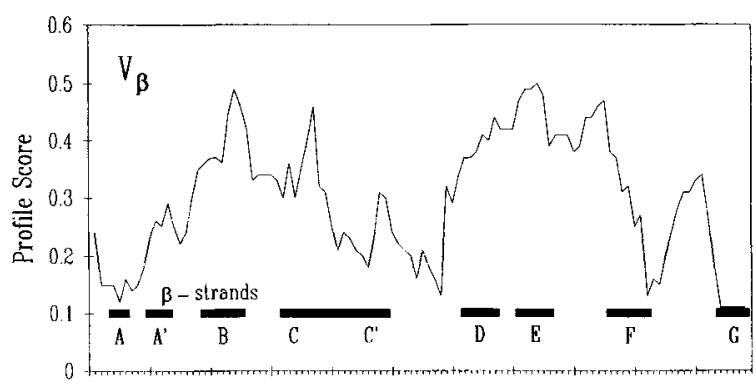

Residue

Fig. 3. 3D-profile of the $\mathrm{V}$ domains of the $5 \mathrm{C} . \mathrm{C} 7$ atomic model. Definition of the $\beta$-strands as in Figure 1.

$\sim 900 \AA^{2}$ for 5C.C7. This surface is higher in size than the lysozyme-antibody interfaces of 690-746 $\AA^{2}$ (Davies et al., 1990). However, this is very similar to the solvent-accessible surface involved in the neuraminidase and the NC41 antibody com- plex of $886 \AA^{2}$ (Davies et al., 1990). The similarity with the neuraminidase-antibody complex can be related to a larger curvature radius of the interaction region of the $\mathrm{MHC}$ and neuraminidase molecules when compared to the corresponding regions of the lysozyme molecule.

Topologically the $5 \mathrm{C} . \mathrm{C} 7$ interface is very irregular; it has a deep depression on one side of the surface and an essentially plane region at the other side (Fig. 6A). The depression built by $\alpha 1, \alpha 2$, and $\beta 3$ (Fig. 6B) is highly complementary with a prominent kink at the helical region of the $\beta-1$ domain of $I-E^{k}$. The $\beta 2$ loop of 5 C.C7 on the other side of the interface is also highly complementary to the $\mathrm{MHC}$ molecule running along the helical region of the $\alpha 1$ domain of I-E ${ }^{k}$ (Fig. 6B). It should be noted that this striking complementarity makes a different positioning of the TCR on the peptide/MHC complex improbable.

The estimate of the peptide/MHC residues in contact with the 5C.C7 molecule shows that seven residues of the MCC peptide and 19 residues of the $I-E^{k}$ molecule are involved in the interaction. Twenty-six residues of the $5 \mathrm{C} . \mathrm{C} 7$ interact with the $\mathrm{MCC}(93-103) / \mathrm{I}-\mathrm{E}^{\mathrm{k}}$ complex (Table 1). Twenty-four residues are located in five of six loops that form the binding site among them, and two residues are placed in the loop linking the $\beta$-strands D-E of $\mathrm{V}_{\alpha}$. The $\beta 1$ loop of 5C.C7 does not interact directly either with the MHC molecule or with the peptide (see Table 1). In $\alpha 1$ and $\alpha 2$ only residues contacting the MHC molecule were found. In contrast, the rest of the loops $(\beta 2, \alpha 3$, and $\beta 3$ ) interact with both the MCC peptide and the MHC molecule.

As predicted by the general TCR/peptide/MHC models (Chothia et al., 1988; Davis \& Bjorkman, 1988; Claverie et al., 1989) and experimental results (Jorgensen et al., 1992a), $\alpha 3$ and $\beta 3$ contact the MCC peptide. However, it is interesting to note that residues of $\alpha 3$ and $\beta 3$ also contact the helices of $I-E^{k}$ mol-

\section{Q1 domain:}

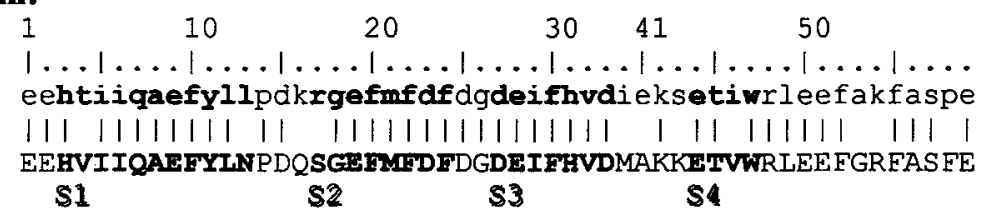

$\begin{array}{cccc}\text { HLA-DR1 : } & \text { EEHVIIQAEFYLNPDSGEMTFDDGDEIFHVDMAKKETVWRLEEFGREASFE } \\ \text { \$1 } & \$ 2 & \$ 3 & \$ 1\end{array}$

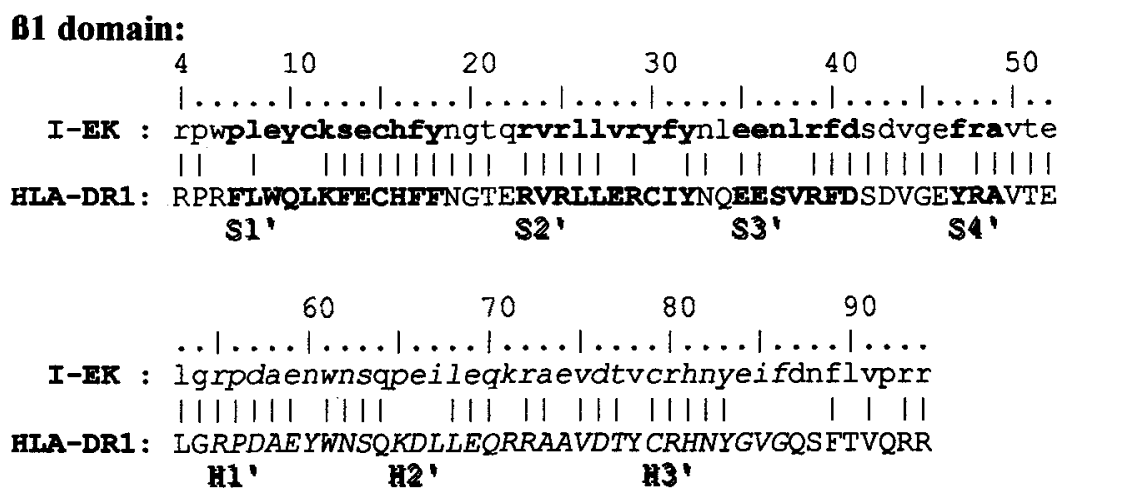

Fig. 4. Alignment between HLA-DR1 and $\mathrm{I}^{-\mathrm{E}^{\mathrm{k}}}$ sequences. Upper part of the figure: $\alpha 1$ domains; lower part: $\beta 1$ domains. In the sequences, letters in bold show the $\beta$-sheets; letters in italics shows $\alpha$-helices as were defined for the HLA-DR1 structure by the Kabsch and Sander (1983) algorithm. 


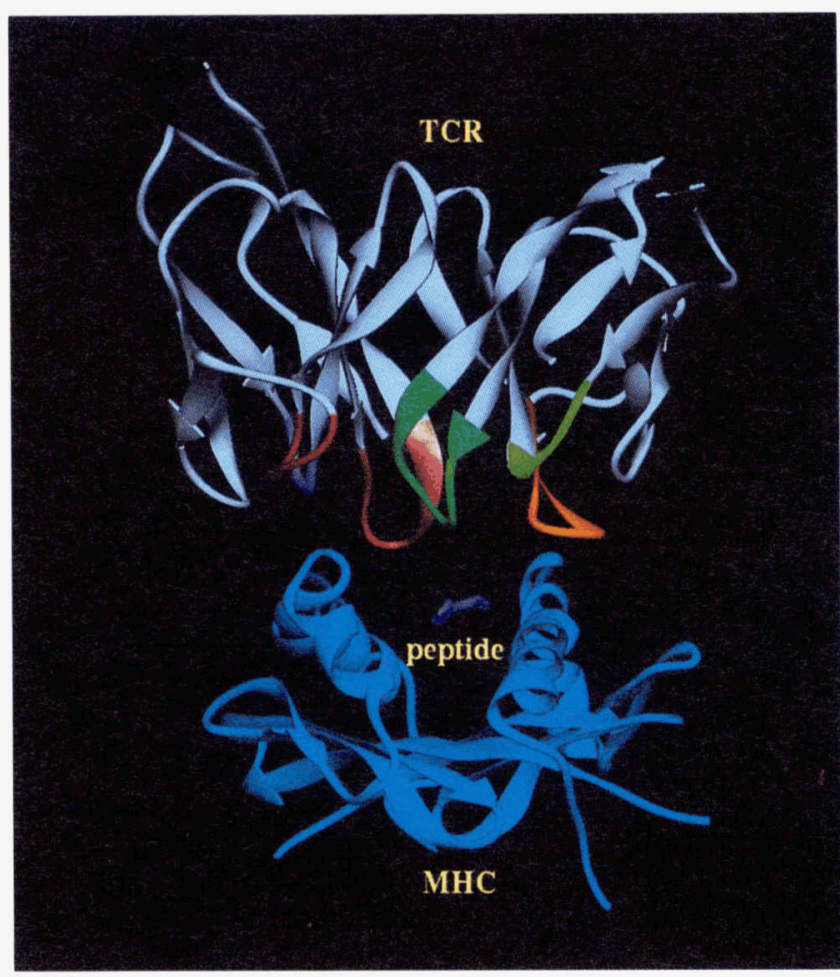

Fig. 5. Ribbon drawings of the $5 \mathrm{C} . \mathrm{C} 7 / \mathrm{MCC}(93-103) / 1-E^{k}$ atomic model. Color code for the molecules: I-E ${ }^{k}$, light blue; MCC(93-103), blue; 5C.C7 framework, white; for the loops that form the antigenbinding site: $\alpha 1$, violet; $\alpha 2$, red; $\alpha 3$, brown; $\beta 1$, light green; $\beta 2$, yellow; $\beta 3$, green

ecule (see Table 1). Taken together these results allow us to propose the way the 5C.C7 molecule recognizes the MCC(93-103)/ $\mathrm{I}-\mathrm{E}^{\mathrm{k}}$ complex in structural terms.

\section{Discussion}

\section{Analysis of the TCR/peptide/MHC interface}

Mapping of the 5C.C7 residues that interact with the MCC (93$103) / I-E^{k}$ structure shows that $\beta 1$ does not contact the peptide/ MHC complex. In addition, the location of $\beta 1$ in the structure suggests that any contact of this loop with the MHC molecule (or the peptide) should imply disruption of some contacts among the residues used to orient the TCR molecule along the peptide/MHC complex (see Kinemage 1). This result suggests that $\beta 1$, at least in this particular complex, does not have a direct role in $\mathrm{MHC}$ recognition.

The residues that interact with the peptide/MHC complex show that $\alpha 1$ and $\alpha 2$ loops are implied only in the recognition of the MHC molecule (see Table 1), whereas $\beta 2, \alpha 3$, and $\beta 3$ contact both the MCC peptide and the I-E $\mathrm{E}^{\mathrm{k}}$ molecule. In T-cell, responses to the MCC peptide presented by $I-E^{k}$ molecules have shown that the TCRs use the same $\mathrm{V}_{\alpha}$ gen segment $\left(\mathrm{V}_{\alpha} 11.1\right.$ [Hedrick et al., 1988; Jorgensen et al., 1992a]), whereas the use of $\mathrm{V}_{\beta}$ does not do so. In $\mathrm{V}_{\beta}$, changes in a single residue in the MCC peptide induce changes in very different patterns of $V_{\beta}$ usage (Jorgensen et al., 1992a). If it is considered that $\alpha 1$ and
Table 1. Residues that interact in the 5C.C7/

MCC(93-103)/I-E $E^{k}$ complex ${ }^{\mathrm{a}}$

\begin{tabular}{|c|c|c|}
\hline $\mathrm{V}_{\alpha}$ & $\begin{array}{l}\text { TCR residues } \\
\text { in contact }\end{array}$ & Peptide/MHC residues in contact \\
\hline \multirow[t]{3}{*}{$\alpha 1$} & $\mathrm{~T}-27$ & $\mathrm{~T}-77(\beta)$ \\
\hline & $\mathrm{R}-29$ & A-73( $(\beta)$, E-74 $(\beta)^{* *}, \mathrm{~T}-77(\beta)^{*}$ \\
\hline & A-30 & $\mathrm{E}-66(\beta)$ \\
\hline \multirow[t]{3}{*}{$\alpha 2$} & $\mathrm{Y}-48$ & Q-64( $\beta)$ \\
\hline & $\mathrm{L}-52$ & $\mathrm{P}-65(\beta), \mathrm{E}-69(\beta)$ \\
\hline & A-53 & $\mathrm{P}-65(\beta)$ \\
\hline \multirow[t]{5}{*}{$\alpha 3$} & E-97 & $\mathrm{K}-99(\mathrm{p})^{* *}$ \\
\hline & S-99 & $\mathrm{T}-77(\beta)^{*}, \mathrm{~V}-78(\beta)^{*}$ \\
\hline & N-100 & $\mathrm{T}-77(\beta), \mathrm{V}-78(\beta), \mathrm{H}-81(\beta), \mathrm{A}-96(\mathrm{p})$ \\
\hline & $\mathrm{T}-101$ & E- $59(\alpha)^{*}$ \\
\hline & N-102 & Q-70( $(\beta)^{*}$, Y-97(p)*, K-99(p), E-74( $\left.\beta\right)^{*}$ \\
\hline \multirow[t]{2}{*}{ Other } & S-68 & $\mathrm{E}-69(\beta)^{*}$ \\
\hline & K-69 & D-76( $\beta)$ \\
\hline \multicolumn{3}{|l|}{$\mathrm{V}_{\beta}$} \\
\hline \multirow[t]{8}{*}{$\beta 2$} & Q-50 & $\mathrm{V}-69(\alpha)$, A-65 $(\alpha)$, Q-100(p)* \\
\hline & N-51 & $\mathrm{A}-72(\alpha), \mathrm{T}-102(\mathrm{p})$ \\
\hline & Q-52 & $\mathrm{K}-71(\alpha), \operatorname{A}-72(\alpha)$ \\
\hline & E-53 & $\mathrm{K}-71(\alpha)^{* *}$ \\
\hline & V-54 & $\mathrm{L}-64(\alpha), \mathrm{A}-68(\alpha), \mathrm{V}-69(\alpha)$ \\
\hline & L-55 & Q-61 $(\alpha)$, L-64 $(\alpha)$ \\
\hline & Q-56 & Q-61 $(\alpha), Y-97(\mathrm{p})^{*}$ \\
\hline & Q-57 & Q-61 $(\alpha)$ \\
\hline \multirow[t]{5}{*}{$\beta 3$} & N-100 & $\mathrm{T}-102(\mathrm{p})^{*}, \mathrm{~K}-103(\mathrm{p})^{*}$ \\
\hline & N-101 & Q-64( $(\beta)^{*}$, A-101(p), T-102(p)*, E-66( $\left.\beta\right)$ \\
\hline & A-102 & $\mathrm{E}-66(\beta), \mathrm{K}-99(\mathrm{p})$ \\
\hline & N-103 & $\mathrm{E}-66(\beta)^{*}$ \\
\hline & S-104 & Q-64 $(\beta)$, E-66 $(\beta)$ \\
\hline
\end{tabular}

${ }^{a}$ An asterisk represents a possible hydrogen bond, two asterisks, a possible salt bridge, no asterisk, a van der Waals contact. Definition of atoms in contact as in Padlan (1994). In parentheses, $\beta$, residues from the $\beta 1$ domain of MHC; $\alpha$, residues from the $\alpha 1$ domain of MHC; $\mathrm{p}$, residues from the antigenic peptide.

$\alpha 2$ are encoded by the $\mathrm{V}_{\alpha}$ gene segment and $\beta 2$ is encoded by the $V_{\beta}$ gen segment (Davis, 1990), the fact that $\alpha 1$ and $\alpha 2$ only contact the MHC molecule explains why the TCRs use the same $\mathrm{V}_{\alpha}$. In addition, the usage of different $\mathrm{V}_{\beta}$ genes is explained by the fact that in contrast to the $\mathrm{V}_{\alpha}$ gene segment, several residues from $\beta 2$ contact the $\alpha$-helix of the $\alpha 1$ domain of ${\mathrm{I}-\mathrm{E}^{\mathrm{k}}}$ as well as several residues of the MCC peptide. Thus, mutation of the peptide would imply changes in the pattern $\mathrm{V}_{\beta}$ gen usage in order to accommodate changes in the peptide.

Although $\beta 2$ contacts both the MCC peptide and the $I-E^{k}$ molecule, there are some residues (from residue 52 to residue 55 , and residue 57) that only interact with the MHC molecule and do not contact the peptide (see Table 1). The region of the $\beta 2$ that interacts with the $\mathrm{I}-\mathrm{E}^{\mathrm{k}}$ molecule is placed mainly at the $\beta$-strand located from residue 53 to residue 58 (see kinemage 1 ). In this interacting region the positions 54 and 55 of 5 C.C7 have two hydrophobic residues, Val and Leu, respectively. These residues form a hydrophobic patch flanked by hydrophilic residues. This hydrophobic patch is in contact with a hydrophobic stretch at the $\alpha$-helix of the MHC molecule: it is formed by Leu-64, Ala-65, Ala-68, and Val-69. In solution, these two hydropho- 


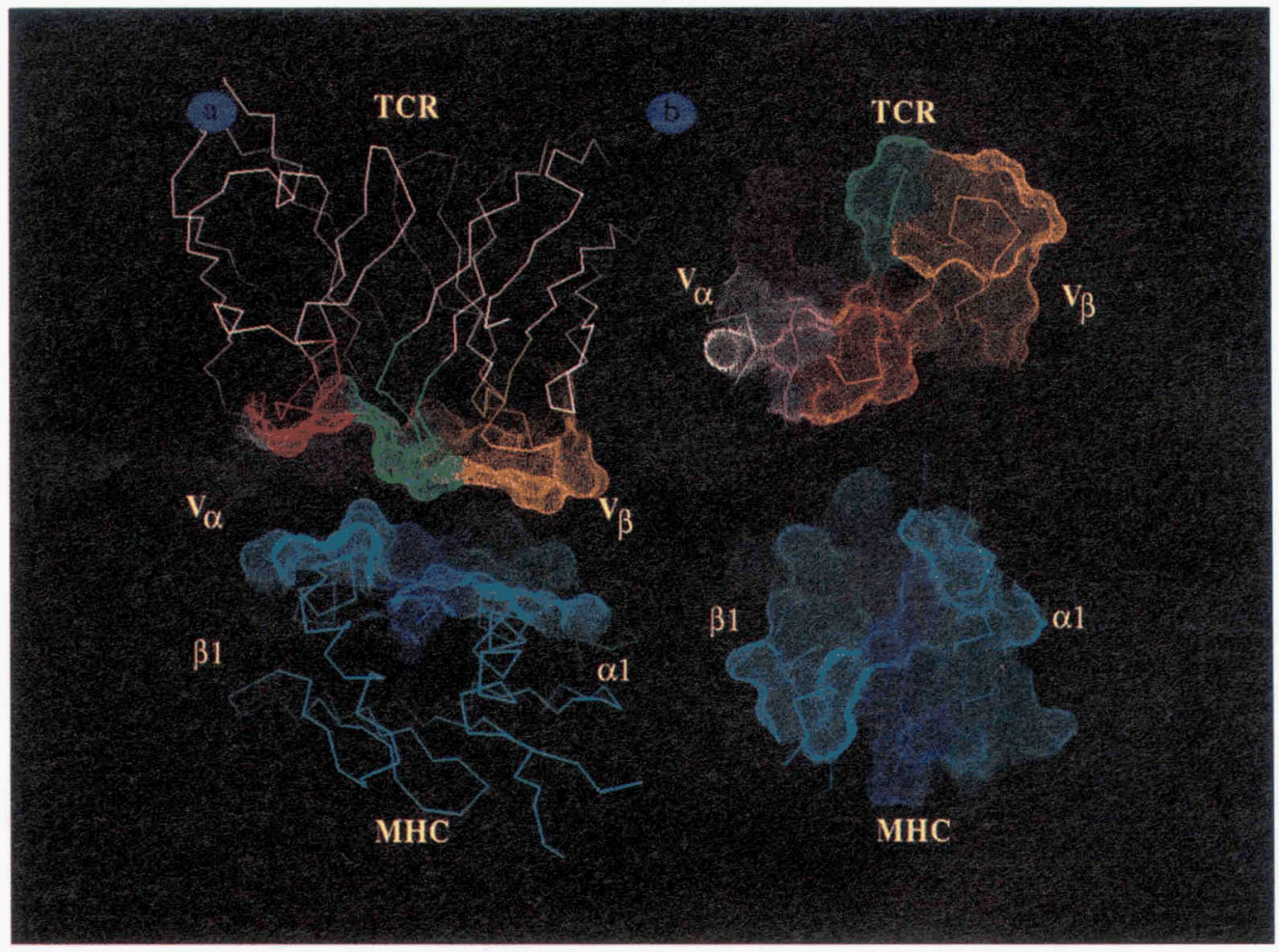

Fig. 6. Solvent-accessible surface generated by the residues that interact in the 5C.C7/MCC(93-103)/I-E ${ }^{k}$ complex. The surface was determined by the Connolly (1983) algorithm with a sphere of 1.7 A diameter. A: View of the complex with the molecules separated by $10 \AA$ to show the surface complementarity. B: Surface view obtained by rotating $90^{\circ}$ the $5 \mathrm{C}$.C7 and MCC $(93-103) / \mathrm{I}-\mathrm{E}^{\mathrm{k}}$ structures with respect to A. Color code as in Figure 5.

bic regions would be exposed to the solvent, which is energetically unfavorable. After complex formation, the hydrophobic regions in both TCR and $\mathrm{I}-\mathrm{E}^{\mathrm{k}}$ are stabilized with one another. This observation suggests that the hydrophobic stretch in the $\alpha 1$ domain of I-E $E^{k}$ is important for recognition by the TCR of the MHC molecule. Supporting this observation, mutations in the ${\mathrm{I}-\mathrm{E}^{\mathrm{k}}}$ sequence within this region avoid the TCR-MHC interaction (Jorgensen et al., 1992b).

Thus, the region placed at the $\beta$-strand of $\beta 2$ on one side of the TCR interface, and $\alpha 1$ and $\alpha 2$ on the other side, would be responsible for the recognition of the MHC molecules. In $\alpha 3$ and $\beta 3$, several residues interact with the peptide and also contact the helical regions of the I- $E^{k}$ molecule. Therefore, $\alpha 3$ and $\beta 3$, together with residues $\beta 2$ that interact with the peptide, would determine the fine recognition of the antigenic peptides in combination with a certain particular MHC molecule.

\section{Implications for $T$-cell recognition}

In the absence of structural experimental information for TCRs, variability analysis of TCR sequences shows that $\alpha 1$ and $\beta 1$ are relatively conserved (Almagro et al., 1995b). This result suggests that $\alpha 1$ and $\beta 1$ would have a different role with respect to $\alpha 2$ and $\beta 2$ in the interaction of the MHC molecules (Almagro et al., 1995b). The general TCR/peptide/MHC interaction models (Chothia et al., 1988; Davis \& Bjorkman, 1988; Claverie et al., 1989), based on the similarity between TCRs and Igs, have not been sufficiently detailed to study the role played by $\alpha 1, \alpha 2, \beta 1$, and $\beta 2$ in the TCR-MHC interaction (Prochnicka-Chalufour et al., 1991).

In the preceding section, several features of the interaction of $\alpha 1, \alpha 2, \beta 1$, and $\beta 2$ were highlighted for the particular case of the 5C.C7/MCC(93-103)/I-E ${ }^{k}$ complex. The results are consistent with the experimental data available for this complex and explain general characteristic of T-cell responses to MCC peptides presented by the $I-E^{k}$ molecule. Thus, this TCR/peptide/MHC interaction model can be useful in providing a structural framework for generating testable hypotheses about the contribution of $\alpha 1, \alpha 2, \beta 1$, and $\beta 2$ to the TCR-MHC interaction.

Overall, the experimental structure for functional domains of MHC class II, $\alpha 1$ and $\beta 1$ are similar to that of class I (Brown et al., 1993). Additionally, comparison of the peptide bond to MHC class I and class II shows that the peptide conformation in both classes of MHCs is extended. The similarity between class I and II MHC molecules and the likeness between the antigenic peptides indicate that the surfaces of peptide/ $\mathrm{MHC}$ molecules presented to the TCRs are roughly similar. If it is considered that the TCR molecules bind the peptide/MHC class I complexes in the same orientation as peptide/class II complexes, as has been suggested by the interaction between superantigen and MHC class I molecules (Bellio et al., 1994) and superantigen class II molecules (Jardetzky et al., 1994), then several general suggestions can be made for the TCR/peptide/MHC interaction. 
Analysis of 5C.C7/MCC $(93-103) / \mathrm{I}-\mathrm{E}^{\mathrm{k}}$ structure showed that $\beta 1$ loop of 5C.C7 does not contact the $\mathrm{MCC}(93-103) / \mathrm{I}-\mathrm{E}^{\mathrm{k}}$ complex. Consistent with our model, experimental data indicate that substitutions in the residues within this region of TCRs do not affect the response to the alloantigens (DiGiusto \& Palmer, 1994). This result agrees also with the low variability found for this loop (Almagro et al., 1995b). In spite of that, experiments with single alanine substitutions on residues of $\beta 1$ indicate that this region may be involved in the peptide-MHC interaction (Bellio et al., 1994; Kang et al., 1994; Lone et al., 1994). The discrepancy found among the experimental results at the $\beta 1$ loop can be explained by: (1) In other TCR molecules, different from 5C.C7, loops shorter in size than $\beta 2$ and $\beta 3$ of $5 C . C 7$ sequence (the loop that blocks the interaction of $\beta 1$ with the $\mathrm{MHC}$ molecule, see Kinemage 1) may allow the $\beta 1$ loop to interact with the helical region of MHC. (2) Analysis of the binding site of 5C.C7 shows that the anchor residue of this loop, His-29, is in a hydrogen bound with Ser-94, a residue placed just before the $\beta 3$ loop. In addition, other residues at the end of the $\beta 1$ loop (Pro-30 and Val-31, see Kinemage 2 ) are in van der Waals contact with the $\beta 3$ loop. This tight interaction among residues of $\beta 1$ and the $\beta 3$ loops might modify the proper positioning of the $\beta 3$ loop to recognize both the peptide and the MHC molecules. Consequently, amino acid substitutions in these residues may abolish the $\mathrm{T}$-cell response. In this sense, a recent report (Katayama et al., 1995) in which $\beta 1$ and $\beta 3$ of 5C.C7 were stitched into the framework of a second TCR indicate that a mutant for the $\beta 3$ loop conferred some but not all of the antigen specificity of 5C.C7, whereas a mutant containing both the $\beta 1$ and $\beta 3$ of $5 \mathrm{C} . \mathrm{C} 7$ recapitulated the antigen specificity of $5 \mathrm{C} . \mathrm{C} 7$ (Katayama et al., 1995). Thus, the above two suggestions to explain the role played by the $\beta 1$ loop in the TCR/peptide/MHC interaction are not mutually exclusive and will have different characteristics in each particular TCR/peptide/MHC system.

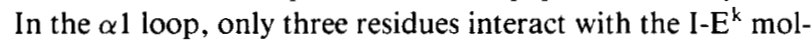
ecule (see Table 1). Experimental data show that the $\alpha 1$ loop of TCR is implied in the recognition of other TCR/peptide/MHC complexes (Nalefski et al., 1992). An analysis of those residues that interact with the $\mathrm{MHC}$ molecule in our model shows that all these residues interact only with nonpolymorphic residues of MHC (Brown et al., 1988). This also is consistent with the variability analysis and suggests that $\alpha 1$ is not a hypervariable region because this interacts with relatively conserved residues of MHC molecules.

Analysis of the residues in $\alpha 2$ that interact with the MHC indicate that its contacts are with the kink region of $\beta 1$ domain of MHC. Comparison between class I and class II molecules show structural differences at this region (Brown et al., 1993). The differences come from a more protuberant kink in the helical region of $\beta 1$ domain of class II molecules when compared with the correspondent region of MHC class I molecules (Brown et al., 1993). This region differing in structure also differs in its pattern of polymorphism. Moreover, even more insertions and deletions are localized at this place within the MHC sequences (Brown et al., 1988). In a compilation of TCR sequences it was shown that five different sizes can be found in the $\alpha 2$ loop of TCRs (Chothia et al., 1988). This fact contrasts with the conserved size of its equivalent loop in Igs, L2 (Chothia et al., 1988). The variability in size of the $\alpha 2$ loop indicates that several conformations are possible for this loop. Together with the structural differences of $\mathrm{MHC}$ molecules at the kink region, this suggests that the interaction between the $\alpha 2$ loop and the kink region of $\mathrm{MHC}$ is critical for distinguishing among different MHCs.

In the $\beta 2$ loop, the residues that interact with the $\mathrm{MHC}$ molecule are placed in a structurally conserved region because it is located on a $\beta$-strand (see Kinemage 1). The region in the $\mathrm{MHC}$ that interacts with the $\beta 2$ loop is also structurally conserved; it is placed in the middle of the $\alpha$-helical region of the $\alpha 1$ domain of MHC. Therefore, one can suggest that at this region only side-chain mutations that conserve the match of the TCR/MHC interaction are allowed. Additionally, the interaction TCRMHC in this region can be modulated by the residues of the peptide, as observed by mapping of the residues at the $5 \mathrm{C} . \mathrm{C} 7 /$ $\mathrm{MCC}(93-103) / \mathrm{I}-\mathrm{E}^{\mathrm{k}}$ complex.

In conclusion, on the basis of the $5 \mathrm{C} . \mathrm{C} 7 / \mathrm{MCC}(93-103) / \mathrm{I}-\mathrm{E}^{\mathrm{k}}$ model, it is possible to suggest a structural role for $\alpha 1, \alpha 2, \beta 1$, and $\beta 2$ in the MHC interaction. These suggestions can be tested by mutational experiments in the absence of structural experimental results for the TCR/peptide/MHC complex in order to understand the structural basis of the recognition mediated by TCRs.

Finally, when this work was submitted for publication, a crystallographic structure of the $\beta$ chain of a TCR $\left(\mathrm{V}_{\beta} 8.2 \mathrm{~J}_{\beta} 2.1 \mathrm{C}_{\beta} 1\right)$ was determined at a resolution of $1.7 \AA$ (Bentley et al., 1995). A comparison of our model with this experimental structure is very valuable for evaluating the structure of the $\beta$ chain of our model. The RMS difference in $\mathrm{C} \alpha$ positions of $\mathrm{V}_{\beta} 8.2 \mathrm{~J}_{\beta} 2.1$ is $1.4 \AA$ with respect to $\mathrm{V}_{\mathrm{L}}$ (compared with framework residues from nine murine $V_{L}$ domains) and $1.9 \AA$ with respect to $V_{H}$ (compared with framework residues from nine murine $V_{H}$ domians) (Bentley et al., 1995). The RMS differences in $\mathrm{C} \alpha$ atoms of the $\beta$ chain of our model with respect to $V_{L}$ and $V_{H}$ of the reference (D1.3) are $1.35 \AA$, and $1.41 \AA$ respectively. In the hypervariable loop $\beta 1$ and $\beta 2$ the comparison between the $5 \mathrm{C} . \mathrm{C} 7$ model and the crystal structure (Fig. 2 of Bentley et al., 1995) shows that the conformations are similar. In the so-called fourth hypervariable region (residues 69-75) the $\mathrm{X}$-ray structure folds toward $\beta 1$ and $\beta 2$ as the homologous region of the $V_{\mathrm{L}}$ domains (Bentley et al., 1995), which are also similar in the equivalent region of the $5 \mathrm{C.C7} \mathrm{V}_{\beta}$ model (see Kinemage 1). The main difference between our model and that structure determined by Bentley et al. (1995) is that the conformation of the crystallographic structure for $\beta 3$ is placed away from $\beta 1$ and $\beta 2$. In the model, $\beta 3$ packs against $\beta 1$ and $\beta 2$. The modeled structure was built for the $\mathrm{V}_{\alpha} / \mathrm{V}_{\beta}$ dimer and $\beta 3$ has a conformation similar to those found in Igs, which is consistent with the proposition of Bentley et al. (1995), establishing that the crystallographic structure does not have the dimer and therefore $\beta 3$ has a conformation that prevents the $V_{\alpha} / V_{\beta}$ dimerization. Thus, all these comparisons show that the differences between the $\mathrm{V}_{\beta}$ chain of the model and the crystallographic structure are small and thus support the good quality of the model.

\section{Materials and methods}

\section{Molecular modeling of 5 C.C7}

All modeling procedures were performed using the program INSIGHT-II (Biosym Technologies, San Diego, California) running on a Silicon Graphic $4 D / 35$. The coordinates of the reported X-ray structure of the D1.3 Fv fragment (entry: IVFA 
[Bath et al., 1990]) obtained from PDB (Bernstein et al., 1977) served as reference in the framework modeling procedure of the 5 C.C7 Fv fragment. The hypervariable loops $\alpha 1, \beta 1$, and $\beta 2$ were modeled with canonical structures. For $\alpha 1$ and $\beta 1$ the coordinates of canonical structure L1 type 1 of the Fab J539 (PDB entry: 2FBJ [T.N. Bhat, E.A Padlan, D.R. Davies, in prep.]) were assigned. For $\beta 2$, the canonical structure type 1 of $\mathrm{H} 2$ of the reference structure (D1.3) was used. Two other hypervariable loops ( $\alpha 3$ and $\beta 3$ ) were modeled starting from the best choice of Ig loops found by using the loop search procedure of the INSIGHT-II package. The database of Ig structures was built with structures collected from the PDB version of October 1993. The X-ray structures found by the loop search procedure and then used as a template to build $\alpha 3$ and $\beta 3$ were the coordinates corresponding to the residues $\mathrm{L} 90-\mathrm{L} 97$ of $\mathrm{KOL}$ (PDB entry: 2FB4 [Marquart et al., 1980]) for $\alpha 3$ and the coordinates corresponding to the residues J95-J101 of $\beta 1312$ (PDB entry: 1IGF [Stanfield et al., 1990]) for $\beta 3$.

The hypervariable loop $\alpha 2$ was modeled as a deletion of two amino acids in the atomic coordinates of $L 2$ in the reference structure (DI.3, see Fig. 1). The gap was closed by doing an energy minimization of the loop fixing all the residues, except the residues adjacent to the gap. After this, the region comprised between the $\beta$-strand $\mathrm{C}^{\prime}$ and $\mathrm{D}$ was subject to molecular dynamic refinement (see below). It is worth mentioning that the possibility of placing the gap after the $\beta$-strand $C^{\prime \prime}$ (an additional $\beta$-strand that includes the residues 53-54, see Fig. 1) was tested. In this latter case, the results of molecular dynamic refinement are locally unstable and the $3 \mathrm{D}$ profile of the region was worse than the chosen model.

\section{Molecular dynamic refinement}

All molecular calculations were carried out using the Discover simulation software package (version 2.7) from Biosym Technologies running on a CRAY Y-MP 4/464 computer. The parameters used were those of the Consistent Valence Force Field (Hagler, 1985). No cross-terms were used in the energy expression and a simple harmonic potential was chosen for the bondstretching terms. Calculations were conducted with a group-based nonbonded cut-off of $10.5 \AA$ imposed over a switching distance of $1.5 \AA$ and updating the nonbonded pair list every 20 time steps using the leap-frog algorithm with a 1-fs time step. All of the hydrogen atoms in the proteins were explicitly modeled. The refinement protocol consisted of successive steps of $5 \mathrm{ps}$ of molecular dynamics each one at $300 \mathrm{~K}$, as follows: (1) refinement of each modeled loop independently; (2) a global refinement of all loops together; (3) a global side-chain refinement of the structures fixing the backbone atoms; (4) a global refinement of the structure tethering $C \alpha$ atoms with a force constant of $60 \mathrm{kcal} / \AA$; (5) a global refinement of all the structures. Before each step, the beginning structure was minimized for 250 steps using the steepest descent method followed by 250 steps using the conjugate gradient method.

Steps 1-3 were archived in vacuo with a constant dielectric of one and the protein having a net total neutral charge. Steps 4 and 5 were performed with the residues charged to simulate pH 7.0 and with a $4-\dot{A}$ thick water shell surrounding the protein to stabilize the charges at the surface of the molecule. In this latter case a linear distance-dependent dielectric constant was used to compensate for the water-vacuo interface. The distance- dependent model together with a water shell surrounding the protein has been shown to achieve a trajectory that better agrees with the crystal structure when compared with a constant dielectric model (Genot \& Kollman, 1992).

The 5C.C7 model refinement consisted of the abovementioned five steps. The $\mathrm{MCC}(93-103) / \mathrm{I}-\mathrm{E}^{\mathrm{k}}$ structure was only subjected to steps 3-5 because of the quality of the initial model. After obtaining a good orientation for the 5C.C7/ MCC $(93-103) / I_{-} E^{k}$ complex, the structure was finally subjected to steps $3-5$.

\section{Acknowledgments}

We thank D. Eisenberg for the 3D profile program, Don C. Wiley and coworkers for kindly providing atomic coordinates for peptide-MHC-II complex, M. Amzel and P. Alzari for valuable comments in early developments of this work, the UNAM supercomputing center for the facilities in the use of the CRAY Y-MP 4/464 computer, and P. Reidy for technical assistance. J.C.A. was supported by DGAPA, grant no. $1 \mathrm{~N}$ 206093. E.V.M. was supported by FOMES, SNI, and CONACyT. E.H. was partially supported by CONACYT.

\section{References}

Ajitkumar P, Geier SS, Kesari KV, Borriello F, Nakagawa M, Bluestonc IA Saper MA, Wiley DC, Nathenson SG. 1988. Evidence that multiple residues on both the $\alpha$-helices of the class 1 MHC molecule are simultaneously recognized by the $T$ cell receptor. Cell 54:47-56.

Almagro JC, Ceceña HA, Vargas-Madrazo E, Lara-Ochoa F. 1995a. Molecular dynamies simulation of an immunoglobulin Fv fragment. 3rd International Perspectives on Protein Engineering, pp. 53-56.

Almagro JC, Zenteno-Cuevas R, Vargas-Madrazo E, Lara-Ochoa F. 1995b Variability analysis of the $\mathrm{T}$-cell receptors using three variability indexes. Int $J$ Pept Protein Res 45:180-186.

Bhat TN, Bentley GA, Fischmann TO, Boulot G, Poljak RJ. 1990. Small rearrangements in structures of Fv and Fab fragments of antibody DI.3 on antigen binding. Nature 347:483-485.

Bcllio M, Lone YC, Calle-Martin O, Malissen B, Abastado JP, Kourilsky $P .1994$. The $V_{3}$ complementary determining region 1 of major histocompatibility complex (MHC) class I-restricted $\mathrm{T}$ cell receptor is involved in the recognition of peptide/MHC I and superantigen/MHC II complex. I Exp Med 179:1087-1097.

Bentley CiA, Boulot G, Karjalainen K, Mariuzza RA. 1995. Crystal struclure of the $B$ chain of a T cell antigen receptor. Science 267:1984-1987.

Bernstein FC, Koetzle TF, Williams GJB, Meyer EF Jr, Brice MD, Rodgers JR, Kennard O, Shimanouchi T, Tasumi M. 1977. The Protein Data Bank: A computer-based archival file for macromolecular structures. $l$ Mol Biol II2:535-542.

Brown JH, Jardetzky IS, Gorga JC, Stern LJ, Urban RG, Strominger JL, Wiley DC. 1993. Three-dimensional structure of the human class 11 histocompatibility antigen HLA-DR I. Nature 364:33-39.

Brown JH, Jardetzky T, Saper MA, Samraoui B, Bjorkman PJ, Wiley DC. 1988. A hypothetical model of the forcign antigen binding site of class If histocompatibility molecules. Nature 332:845-850.

Chothia C, Boswell DR, Lesk AM. 1988. The outline structure of the T-cell $\alpha: \beta$ receptor. $E M B O J 7: 3745-3755$.

Chothia C, Lesk AM. 1987. Canonical structures for the hypervariable re gions of immunoglobulin. $J$ Mol Biol 196:901-917.

Chothia C, Lesk AM, Gherardi E, Tomlinson IM, Walter G, Marks JD, Llewelyn MB, Winter G. 1992. Structural repertoire of the human $\mathrm{VH}$ segments. $J$ Mol Biol 227:799-817.

Chothia C, Lesk AM, Tramontano A, Levitt M, Smith-Gill SJ, Air G, Sheriff S, Padlan EA, Davics D, Tulip WR, Colman PM, Spinelli S, Alzari PM, Poljak RJ. 1989. Conformations of immunoglobulin hypervariable regions. Nuture (Lond) 342:877-883.

Claverie JM, Prochnicka-Chalufour A, Bougueleret 1.. 1989. Implications of a Fab-like structure for T-cell receptor. Immunol Today 10:10-14.

Connolly ML. 1983. Solvent-accessible surfaces of proteins and nucleic acids. Science 221:709-713.

Davies DR, Padlan EA, Sheriff S. 1990. Antibody-antigen complexes. Annu Rev Biochem 59:439-473.

Davis MM. 1990. T cell receptor gene diversity and selection. Anmu Rev Biochem 59:475-496. 
Davis MM, Bjorkman PJ. 1988. T-cell antigen receptor genes and T-cell recognition. Nature 334:395-402.

DiGiusto DL, Palmer E. 1994. An analysis of sequence variation in the $\beta$ chain framework and complementarity determining regions of an alloreactive T cell receptor. Mol Immunol 31:693-699.

Ganju RK, Smiley ST, Bajorath J, Novotny J, Reinherz EL. 1992. Similarity between fluorescein-specific T-cell receptor and antibody in chemical details of antigen recognition. Proc Natl Acad Sci USA 89:11552-11556.

Genot J, Kollman PA. 1992. Molecular dynamics studies of a DNA-binding protein: 2. An evaluation of implicit and explicit solvent models for the molecular dynamics simulation of the Escherichia coli trp repressor. Protein Sci $1: 1185-1205$.

Hagler AT. 1985. Theoretical simulation of conformation, energetics, and dynamics of peptides. In: Meienhofer J, ed. Conformation in biology $\&$ drug design, the peptides, vol 7. New York: Academic Press. pp 213228.

Hedrick SM, Engel I, McElligot DL, Fink PJ, Hsu ML, Hansburg D, Matis A. 1988. Selection of amino acid sequences in the $\beta$ chain of $T$ cell antigen receptor. Science 239:1541-1544.

Jardetzky TS, Brown JH, Gorga JC, Stern LJ, Urban RG, Chi Yi, Stauffacher C, Strominger JL, Wiley DC. 1994. Three-dimensional structure of a human class II histocompatibility molecule completed with superantigen. Nature 368:711-718.

Jorgensen JL, Esser U, Fazekas de St Groth B, Reay PA, Davis MM. 1992a. Mapping T-cell receptor-peptide contacts by variant peptide immunization of single-chain transgenies. Noture 355:224-230.

Jorgensen JL, Reay PA, Ehrich EW, Davis MM. 1992b. Molecular components of T-cell recognition. Annu Rev Immunal 10:835-873.

Kabat EA, Wu TT, Perry HM, Gottesman KS, Foeller C. 1991. Sequences of proteins of immunological interest, 5th ed. Bethesda, Maryland: U.S. Department of Health and Human Services, Public Health Service, National Institutes of Health, NIH Publication No. 91-3242.

Kabsch W, Sander C. 1983. Dictionary of protein secondary structure: Pattern recognition of hydrogen-bonded and geometrical features. Biopolymer 22:2577-2637.

Kang J, Chambers C, Pawling J, Scott C, Hozumi N. 1994. Conserved amino acids residues in the complementarity determining region 1 of the TCR $\beta$-chain are involved in the recognition of conventional Ag and Mls- $1 \mathrm{su}-$ perantigen. $J$ Mol Immunol 152:5305-5317.

Katayama CD, Eidelman FJ, Dunan A, Hooshmand, Hedrick SM. 1995. Predicted complementary determining regions of the $\mathrm{T}$ cell antigen receptor determine antigen specificity. EMBO J 14:927-938.

Lone YC, Bellio M, Prochnicka-Chalufour A, Ojcius DM, Boissel N, Ot- tenhoff TMH, Klausner RD, Abasto JP, Kourilsky P. 1994. Role of the CDR1 region of the TCR $\beta$ chain in the binding to purified MHC-peptide complex. Int Immunol 6:1561-1565.

Lüthy R, Bowie JV, Eisenberg D. 1992. Assessment of protein models with three-dimensional profiles. Nature 356:83-85.

Marquart M, Deisenhofer J, Huber R. 1980. Crystallographic refinement and atomic models of the intact immunoglobulin molecule $\mathrm{Kol}$ and its antigen-binding fragment at $3.0 \AA$ and $1.9 \AA$ resolution. $J \mathrm{Mol} \mathrm{Biol} / 4 \mathrm{I}$ : 369-391.

Nalefski EA, Kasibhatla S, Rao A. 1992. Functional analysis of the binding site on the T cell receptor $\alpha$ chain. J Exp Med 175:1553-1563.

Novotny J, Tonegawa S, Saito H, Kranz DM, Eisen H. 1986. Secondary, tertiary, and quaternary structure of T-cell-specific immunoglobulin-like polypeptide chains. Proc Natl Acad Sci USA 83:742-746.

Padlan EA. 1994. The anatomy of the antibody molecule. Mol Immunol $31: 169-193$

Patten PA, Rock EP, Sonoda T, Fazekas de St Groth B, Jorgensen JL, Davis MM. 1993. Transfer of putative complementarity-determining region loops of $\mathrm{T}$ cell receptors $\mathrm{V}$ domains confers toxin reactivity but not peptide/MHC specificity. J Immunol 150:2281-2294.

Poljak R J, Amzel LM, Avey HP, Chen BL, Phizacherley RP, Saul F. 1973. Three-dimensional structure of the $\mathrm{Fab}^{\prime}$ fragment of a human immunoglobulin at 2.8-Ä resolution. Proc Natl Acad Sci USA 70:3305-3310.

Prochnicka-Chalufour A, Casanova JL, Avrameas S, Claverie JM, Kourilsky P. 1991. Biased amino acids distribution in regions of the $T$ cell receptors and MHC molecules potentially involved in their association. in Immunol 3:853-864.

Stanfield RL, Fieser TM, Lerner RA, Wilson IA. 1990. Crystal structures of an antibody to a peptide and its complex with peptide antigen at $2.8 \AA$ Science 248:712-719.

Stern LJ, Brown JH, Jardetzky TS, Gorga JC, Urban RG, Strominger JL, Wiley DC. 1994. Crystal structure of the human class II MHC protein HLA-DR 1 completed with an antigenic peptide from influenza virus. $\mathrm{Na}$ ture 368:215-221,

Tramontano A, Chothia C, Lesk AM. 1990. Framework residue 71 is a major determinant of the position and conformation of the second hypervariable region in the $\mathrm{V}_{\mathrm{H}}$ domains of immunoglobulins. J Mol Biol 215: $175-182$.

Wilson IA, Stanfield RL. 1993. Antibody-antigen interactions. Curr Opin Struct Biol 3:113-118.

Wu TT, Kabat EA. 1970. An analysis of the sequences of the variable regions of Bence Jones proteins and myeloma light chains and their im plications for antibody complementarity. J Exp Med 132:211-250. 